\title{
ESTUDIO DE UNA CELDA DE COMBUSTIBLE ORGÁNICO ACOPLADA A UN PROCESO DE TRATAMIENTO BIOLÓGICO DE AGUAS RESIDUALES
}

\section{STUDY OF A CELL OF ORGANIC FUEL COUPLED TO A PROCESS OF BIOLOGICAL TREATMENT OF WASTEWATER}

\author{
MSc. Jose Carlos Galindo González \\ Fundación Universitaria del Área Andina, Facultad de Ingeniería y Ciencias Básicas, \\ Grupo de Investigación e Innovación en Ingeniería Civil - GINC. \\ Trans 22 Bis \# 4 -105, Sector Callejas, Valledupar, Cesar, Colombia \\ Teléfono: (+57)(5) 5897879 \\ E-mail: jgalindo22@areandina.edu.co
}

\begin{abstract}
Resumen: Una celda de combustible orgánico (CCO) fue acoplada a un reactor biológico utilizado para el tratamiento de aguas residuales con el objeto de evaluar aspectos que afectan la eficiencia de generación de energía eléctrica del arreglo. El estudio fue realizado en dos fases. En la inicial, se generó $49.47 \mathrm{~mW} / \mathrm{m} 2$, alcanzando una remoción de materia orgánica, medida como mg/L DQO, del $77.44 \%$. En la segunda fase se ensayaron varias configuraciones. Valores máximos de voltaje $(800 \mathrm{mV})$ y corriente $(4.2$ $\mathrm{mA}$ ) se alcanzaron usando como electrolito una solución $\mathrm{NaCl}$ de $4.0 \mathrm{M}$. Las demás evaluaciones se realizaron variando la relación de áreas ánodo/cátodo, usando un catalizador en la cámara de reducción, y variando la resistencia del circuito externo. Con los resultados se demostró que es posible generar energía eléctrica en una CCO utilizando agua residual como combustible. También que los aspectos analizados inciden en los niveles de generación de energía eléctrica.
\end{abstract}

Palabras clave: Celda de combustible orgánico (CCO), reactor biológico, tratamiento biológico de aguas residuales.

\begin{abstract}
An organic fuel cell was attached to a bioreactor used to wastewater treatment in order to determine the aspects that may affect electric energy generation efficiency. Research had two phases. The first generated $49.47 \mathrm{~mW} / \mathrm{m} 2$ while removing $77.44 \%$ of organic matter, measure as COD. At the second stage the system reached up values of voltage and current of $800 \mathrm{mV}$ and $4.2 \mathrm{~mA}$ using an electrolyte concentration of $\mathrm{NaCl}$ 4.0M solution. The rest of the second phase were testing different anode/cathode ratio area, a catalyst dissolved in reduction camera, and different external circuit resistance. It was possible for OFC to electricity generated using wastewater as fuel. The results indicated that factors analyzed influenced device electricity generation capacity.
\end{abstract}

Keywords: Organic fuel cell (OFC), bioreactor, biological wastewater treatment.

\section{INTRODUCCIÓN}

Las celdas de combustible han sido utilizadas para producir energía eléctrica a partir de diferentes compuestos orgánicos (Logan y Elimelech, 2012; Minteer, 2017; Santoro et al., 2017; Sieniutycz y
Jezowski, 2017). En la actualidad, esta práctica está encaminada al uso de aguas residuales como combustible, donde microorganismos encargados de degradar la materia orgánica transfieren electrones a un ánodo y estos pasan a través de un circuito externo, creando un flujo de electrones que 
no es más que una corriente eléctrica (Pasternak et al., 2017; Roustazadeh Sheikhyousefi et al., 2017; YE Santafé, BD Chaparro, 2013). El uso de estos dispositivos acoplados al tratamiento de aguas residuales brinda la oportunidad de generar energía eléctrica a muy bajo costo, constituyéndose como un proceso autosostenible que puede llevar consigo la disminución de la cantidad de lodos a disponer, la no utilización de combustibles fósiles y por ende un bajo impacto ambiental. En un futuro, este mecanismo de fácil implementación podría brindar la posibilidad de suplir las demandas energéticas para comunidades apartadas, convirtiéndose en un sistema no centralizado de generación de energía eléctrica (LOT Tellez, MPS Delgado, 2017).

\section{MATERIALES Y METODOLOGÍA}

\subsection{Reactor biológico y celda de combustible}

El reactor biológico configurado como se muestra en la figura 1 fue elaborado con láminas de acrílico de $3 \mathrm{~mm}$ de espesor. Estaba divido en 4 secciones que se describen a continuación:

- Sección 1: Electrolito ( $\mathrm{NaCl}$ - 1.0 M). Contenía al cátodo y tenía un volumen de 2.25 litros $(5 \mathrm{~cm}$ de ancho, $20 \mathrm{~cm}$ de largo y $25 \mathrm{~cm}$ de alto, con un borde libre de $5 \mathrm{~cm}$ ).

- Sección 2: Reactor biológico (cámara de oxidación). En él se encontraban los lodos activados, obtenidos de una planta de tratamiento de aguas residuales del Municipio de Tenjo, Departamento de Cundinamarca. En esta sección, se dosificaba el agua residual $(\mathrm{Q}=7.0 \mathrm{~mL} / \mathrm{min}$, $\mathrm{TRH}=12$ horas) y tenía un volumen de 6 litros (15 cm de ancho, $20 \mathrm{~cm}$ de largo y $25 \mathrm{~cm}$ de alto, con un borde libre de $5 \mathrm{~cm}$ ).

- Sección 3: Cámara de transferencia de protones. En esta se vertía el efluente de la cámara de oxidación.

- Sección 4: Cámara de reducción. En esta sección, que contenía el cátodo, se encontraba una solución de cloruro férrico $\left(\mathrm{feCl}_{3}-0.1 \mathrm{M}\right)$, que se utilizó como agente aceptor final de electrones. $\mathrm{Su} \mathrm{pH}$ fue modificado con el objetivo de que el hierro férrico se precipitara. Para esto se adicionó Bicarbonato de Sodio. Adicional a esto, se colocaron en el fondo láminas de acrílico inclinadas. Tenía un volumen de 6 litros y contaba con un aireador con el que se hacia el suministro del oxígeno y ayudaba a que el hierro férrico permaneciera en suspensión.

\subsection{Circuito eléctrico}

Conformado por los electrodos (ánodo y cátodo) y el cable que los unía. Se utilizaron barras de grafito $(15.8 \mathrm{~cm}$ de alto, $3.8 \mathrm{~cm}$ de ancho y $1.2 \mathrm{~cm}$ de espesor) con un área superficial de $0.01637 \mathrm{~m}^{2}$.

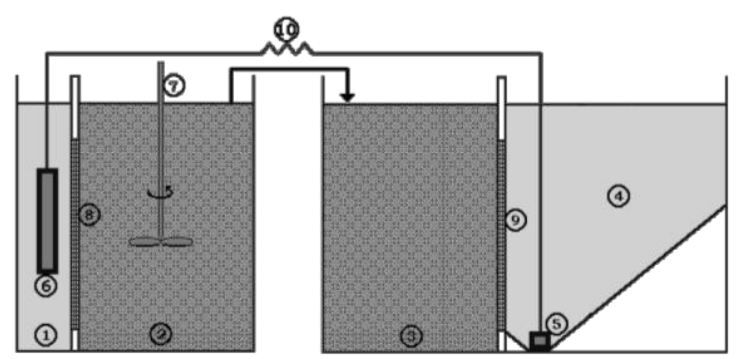

Fig. 1. Esquema del reactor biológico utilizado como celda de combustible orgánico. La sección 1 contiene el electrolito, la sección 2 representa la cámara de oxidación, la sección 3 representa la cámara de transferencia de protones, la sección 4 representa la cámara de reducción. El circuito estaba conformado por 6, 5 y 10 que representan al ánodo, cátodo, el cable de cobre más la resistencia, respectivamente. El numero 7 representa el mezclador y los números 8 y 9 las membranas de intercambio iónico, en este caso, aniónica y catiónica respectivamente. Fuente: El autor.

Se hizo la limpieza de los electrodos introduciéndolos en una solución de $\mathrm{NaOH}-1.0 \mathrm{~N}$ durante 24 horas y luego en una solución de $\mathrm{HCl}$ $1.0 \mathrm{~N}$ durante el mismo tiempo. Después se colocaban en agua destilada y se secaban al horno durante 30 minutos a una temperatura de $60{ }^{\circ} \mathrm{C}$. El objetivo de este procedimiento era eliminar cualquier residuo de metal o biomasa que pueda adherirse a ellos.

La conexión de los electrodos se hizo con un cable de cobre No. 12 AWG, que se insertó en los electrodos haciendo un orificio en el medio de su superficie.

En la primera fase de operación se colocó una resistencia de $1000 \Omega$ entre los electrodos y los valores de voltaje se midieron con un multímetro marca EBC-96820/UT30C. En la segunda fase se utilizó una caja de resistencias con un selector, la cual permitía seleccionar una resistencia de 1000 , $500,200,100,50$ o $10 \Omega$. Las lecturas de voltaje y corriente se determinaron bajo dos condiciones: circuito abierto y circuito cerrado.

\subsection{Membranas de intercambio iónico}

Se emplearon membranas de intercambio iónico de 
la serie Ionac $^{\circledR}$ Ion Exchange Membranes. Se utilizó una membrana aniónica (MA - 3475, área de $192 \mathrm{~cm}^{2}$ y espesor de $1.5 \mathrm{~mm}$ ) y una membrana catiónica (MC - 3470, área de $192 \mathrm{~cm}^{2}$ y espesor de $1.5 \mathrm{~mm}$ ). La primera estaba ubicada entre el electrolito y la cámara de oxidación (sección 1 y sección 2 respectivamente). La segunda membrana se colocó entre la cámara de transferencia de protones y la cámara de reducción (sección 3 y sección 4 respectivamente) (Fig. 1).

\subsection{Agua residual}

El agua residual fue preparada en laboratorio (Tabla. 1). Pasado cuatro días el agua perdía en gran cantidad sus características iniciales por lo que cada 4 días se preparaba un nuevo sustrato y se lavaba todo el sistema hidráulico con el fin de no alterar las condiciones iniciales. Sus características fueron: $\mathrm{DQO}=283.73 \pm 7.46 \mathrm{mg} / \mathrm{L}, \mathrm{pH}=6.89 \pm$ 0.05 y Temperatura $=18 \pm 0.7^{\circ} \mathrm{C}$.

Tabla 1: Componentes y cantidades del agua residual

\begin{tabular}{lc}
\hline \multicolumn{1}{c}{ Componentes } & $\begin{array}{c}\text { Cantidad } \\
(\mathbf{m g} / \mathbf{L})\end{array}$ \\
\hline Bicarbonato de sodio & 120 \\
Hidrógeno fosfato de sodio & 2.7 \\
Cloruro férrico hexahidratado & 4.0 \\
Cloruro de potasio & 3.2 \\
Sulfato de magnesio heptahidratado & 2.4 \\
Papel higiénico & 3.2 \\
Jabón en polvo & 2.4 \\
Urea & 24 \\
Gelatina & 48 \\
Almidón & 240 \\
Leche en polvo & 144 \\
\hline
\end{tabular}

Fuente: El autor.

\subsection{Equipo adicional}

- Bomba dosificadora: Para dosificar el agua residual se utilizó una bomba peristáltica de flujo continuo y un control de velocidad. Se utilizaron mangueras de silicona para las conexiones hidráulicas.

- Mezclador: Se utilizó para mantener el reactor biológico en condiciones de mezcla completa (200 r.p.m.).

- Aireador: La inyección del oxígeno en la cámara de reducción se hizo por medio de una piedra difusora conectada a un aireador.

- Tanque de almacenamiento del efluente: Se colocó una salida desde la cámara de reducción (a $5 \mathrm{~cm}$ del borde) conectada a un tanque para recolectar el efluente. A esta agua se le practicaba ensayos de laboratorio (DQO-Standard Methods, 5220D) para determinar la materia orgánica remanente luego del tratamiento.

- Sistema de adquisición de datos: Para cuantificar el voltaje y la corriente en la segunda fase de operación se implementó un sistema de adquisición de datos National Instruments Corporation y el software LabVIEW.

La Figura 2 muestra el montaje inicial junto con el sistema de adquisición de datos implementados para la segunda fase de operación.

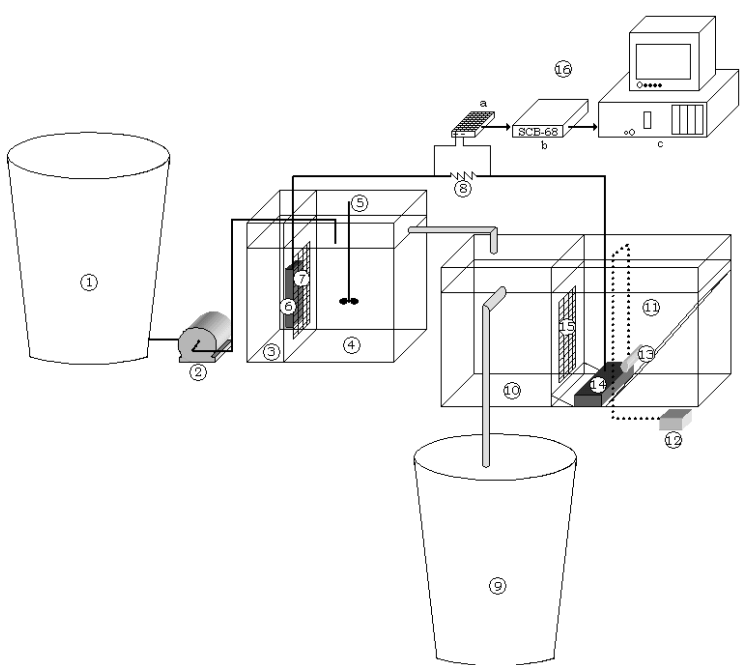

Fig. 2. Esquema y detalle del montaje inicial y del sistema de adquisición de datos implementados para la segunda fase de operación. 1.-Tanque de Agua residual, 2.-Bomba Peristáltica, 3.Electrolito, 4.-Cámara de Oxidación, 5.Mezclador, 6.-Anodo, 7.-Membrana Aniónica, 8.Circuito eléctrico, 9.-Tanque para almacenar el efluente, 10.-Cámara de Transferencia de

Protones, 11.-Cámara de Reducción, 12.-Aireador, 13.-Piedra Difusora, 14.-Cátodo, 15.-Membrana Catiónica y 16.-Sistema de Adquisición de datos. a) Caja de resistencias, b) Bloque Conector y c) Computador. Fuente: El autor.

\section{RESULTADOS Y ANÁLISIS}

\subsection{Fase I}

En esta etapa, se evaluó la eficiencia del sistema en cuanto a la remoción de materia orgánica (medida como mg/L de DQO), la corriente y potencia 
generada y la variación del $\mathrm{pH}$ en las cámaras de oxidación y de transferencia de protones.

Los microorganismos utilizados para el tratamiento del agua residual se reprodujeron rápidamente durante los primeros días de operación, lo cual se evidenció por el aumento en la turbiedad en la cámara de oxidación y en la presencia de lodos en el efluente. A partir del día 21 la presencia de lodos en el efluente disminuyó al igual que la turbiedad en la cámara de oxidación, lo cual indicó que se alcanzó la fase estacionaria del crecimiento bacterial. Los parámetros de operación de la Fase I se presentan en la Tabla 2.

Tabla 2: Parámetros de operación de la Fase I

\begin{tabular}{lc}
\hline Parámetro & Valor \\
\hline Caudal & $7 \mathrm{~mL} / \mathrm{min}=10 \mathrm{~L} /$ día \\
DQO & $283.73 \pm 7.46 \mathrm{mg} / \mathrm{L}$ \\
TRH & $0.5 \mathrm{~d}=12 \mathrm{~h}$ \\
R circuito & $1 \mathrm{k} \Omega=1000 \Omega$ \\
Concentración electrolito & $1.0 \mathrm{M}$ \\
Concentración FeCl3 & $0.1 \mathrm{M}$ \\
\hline
\end{tabular}

\section{Fuente: El autor.}

Entre los días 11 y 21 el sistema pasó de una remoción de materia orgánica del 27 al $54 \%$, llegando hasta un valor máximo del $77.44 \%$ para los últimos 11 días (Fig. 3).

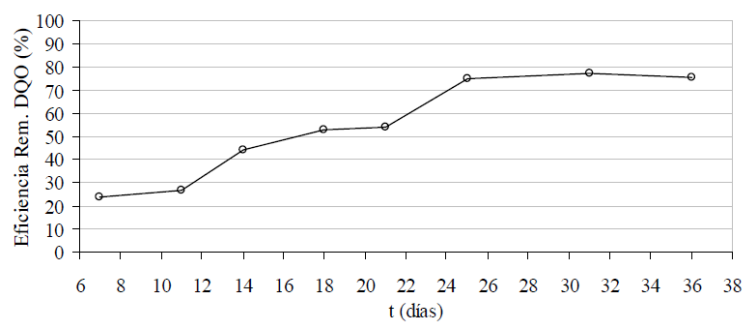

Fig. 3. Eficiencia en la remoción de materia orgánica. Fuente: El autor.

Durante los primeros 21 días el sistema no generó una corriente superior a $0.2 \mathrm{~mA}$, en comparación con los últimos 15 días donde se generaron corrientes entre 0.805 y $0.86 \mathrm{~mA}$ (Fig. 4). Este comportamiento se debió a que durante los primeros 21 días (fase de crecimiento exponencial) los microorganismos requieren gran parte de la energía disponible, y que obtienen del sustrato, para alcanzar su desarrollo, representado por sus procesos de reproducción, crecimiento, movimiento y manutención.

El resultado supone que los niveles de energía obtenidos en una celda de combustible orgánico dependen de la etapa en que se encuentre el desarrollo microbial del sistema; es decir, un sistema en proceso de aclimatación generará menos corriente que aquel que se encuentre en una etapa o fase madura.

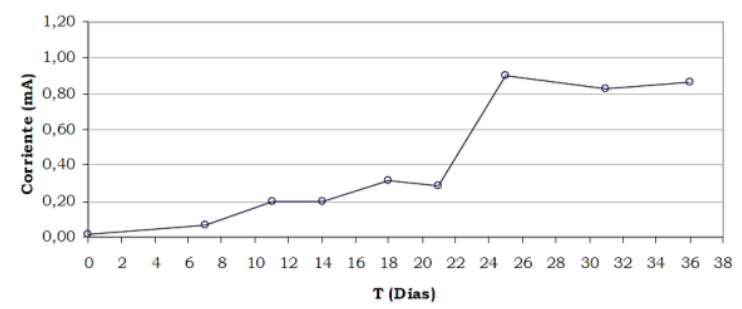

Fig. 4. Variación de corriente fase I. Fuente: El autor.

Se relacionaron los valores de corriente generada y la eficiencia en la remoción de materia orgánica, obteniendo que se ajustan a un comportamiento exponencial con una correlación de los datos de 0.87 y un error típico del $8.17 \%$ (Fig. 5).

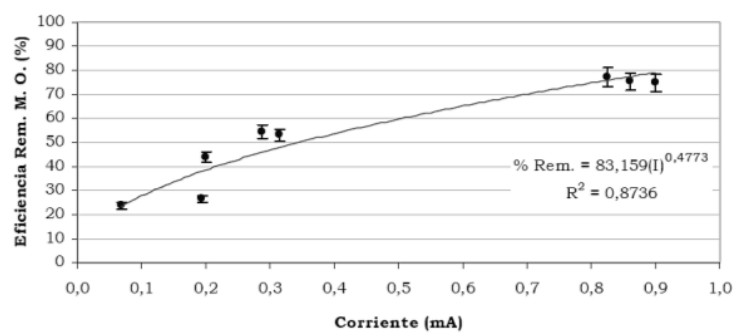

Fig. 5. Relación entre corriente producida y eficiencia en la remoción de materia orgánica medida como $\mathrm{mg} / \mathrm{L}$ de DQO, fase I. Fuente: El autor.

Se alcanzó un valor máximo de potencia de 49.47 $\mathrm{mW} / \mathrm{m}^{2}$ (Fig. 6), que al ser comparados con los obtenidos en otras investigaciones resulta ser superior, puesto que el área de los electrodos (expresada en metros cuadrados) de esta investigación es superior, lo cual hace que el valor de la potencia sea amplificado en el cálculo.

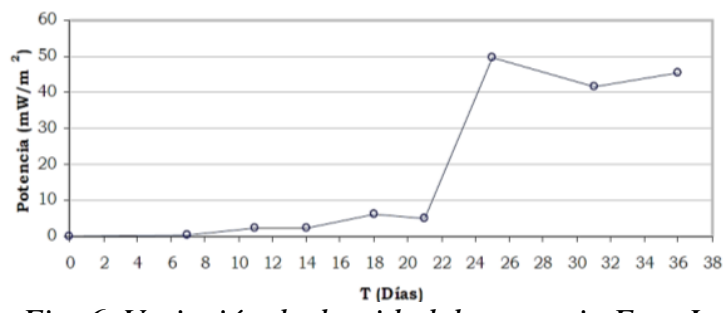

Fig. 6. Variación de densidad de potencia Fase I. Fuente: El autor.

Se determinaron los valores de $\mathrm{pH}$ en la cámara de oxidación y de transferencia de protones, resultando ser los de la primera ligeramente inferiores a los de la segunda (Fig. 7). 


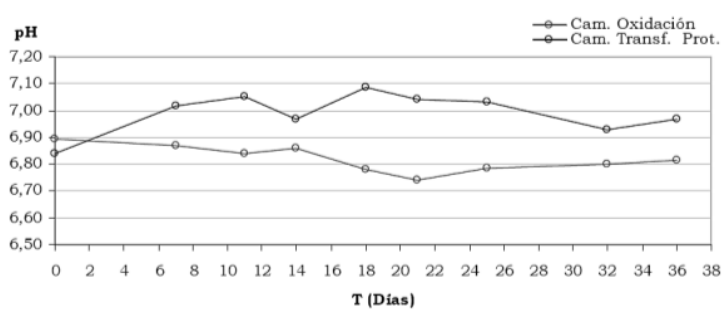

Fig.7. Variación de pH en cámaras de oxidación y de transferencia de protones fase I. Fuente: $E l$ autor.

Aunque en teoría el agua que contenían ambas cámaras era la misma, esta diferencia evidencia el propósito para el cual se colocó la membrana catiónica. La función de ésta en el sistema era la de permitir el paso de los $\mathrm{H}^{+}$generados en la cámara de oxidación durante el proceso de oxidación de la materia orgánica por parte de los microorganismos. Con esto, se obtenía el equilibrio de cargas entre la cámara de oxidación y la de reducción.

La ecuación (1) representa el proceso aerobio de degradación de la materia orgánica (Metcalf \& Eddy, 2013). El circulo muestra que como producto del proceso se generan hidronios $\left(\mathrm{H}^{+}\right)$, los cuales son liberados en la cámara de oxidación y transferidos a la de reducción por medio de la membrana catiónica.

$\frac{1}{50} \mathrm{C}_{10} \mathrm{H}_{19} \mathrm{O}_{3} \mathrm{~N}+\frac{9}{25} \mathrm{H}_{2} \mathrm{O} \longrightarrow \frac{9}{25} \mathrm{CO}_{2}+\frac{1}{50} \mathrm{NH}_{4}^{+}+\frac{1}{50} \mathrm{HCO}_{3}+\underset{\mathrm{H}^{+}}{\stackrel{H}{ }+\mathrm{e}}$

Esto demuestra que es indispensable la ubicación de la membrana catiónica en una celda de combustible, ya que ayuda a la migración de cationes, equilibrando las cargas en el sistema derivadas del transporte de electrones efectuado a través del circuito externo.

Luego de la evaluación de los datos de la fase I, se siguió operando el sistema en las mismas condiciones por espacio de 45 días; luego de este tiempo se dio inicio a la fase II cuyos resultados se muestran continuación.

\subsection{Fase II}

Con la realización de esta etapa se buscó determinar la incidencia que tiene en la generación de energía eléctrica por parte de las celdas de combustible orgánico ciertos parámetros operacionales.

Los parámetros adoptados para la operación de la Fase II se presentan en la Tabla 3.
Tabla 3: Parámetros de operación de la Fase II

\begin{tabular}{lc}
\hline Parámetro & Valor \\
\hline Caudal & $7 \mathrm{~mL} / \mathrm{min}=10 \mathrm{~L} /$ día \\
DQO & $283.73 \pm 7.46 \mathrm{mg} / \mathrm{L}$ \\
TRH & $0.5 \mathrm{~d}=12 \mathrm{~h}$ \\
R circuito & $200 \Omega$ \\
Concentración electrolito & $1.0 \mathrm{M}^{*}$ \\
Concentración $\mathrm{FeCl} 3$ & $0.1 \mathrm{M}^{*}$ \\
\hline
\end{tabular}

* Valores iniciales, posteriormente fueron variados según cambios hechos a la configuración inicial.

Fuente: El autor.

Se hicieron múltiples diluciones del afluente y se alimentó el sistema durante 1 día con cada una de éstas (Tabla 4). Se determinó el valor máximo de corriente producido en cada día al cerrar el circuito con la resistencia de $200 \Omega$.

Tabla 4: Porcentaje de dilución del agua residual

\begin{tabular}{cc}
\hline Dilución & DQO $(\mathbf{m g} / \mathbf{L})$ \\
\hline 100 & 287 \\
60 & 172.2 \\
50 & 143.5 \\
30 & 86.1 \\
25 & 71.8 \\
10 & 28.7 \\
\hline \multicolumn{2}{c}{ Fuente: El autor. }
\end{tabular}

Los datos obtenidos fueron ajustados mediante una regresión lineal (Fig. 8) con una correlación de 0.9188 y un error típico del $4.93 \%$.

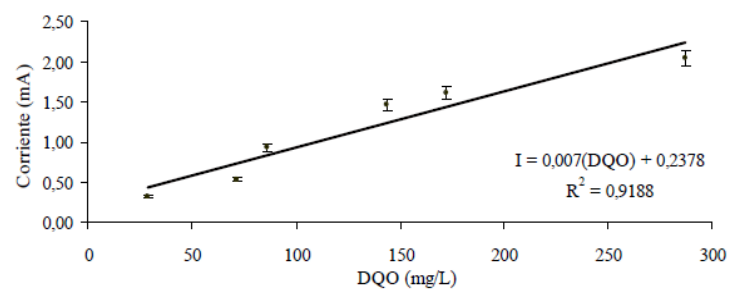

Fig. 8. Relación entre diferentes concentraciones de DQO del agua residual y corriente generada.

Fuente: El autor.

Con este procedimiento se evaluó el efecto que tiene la carga orgánica respecto a la generación de energía. Se concluye que, a mayor carga orgánica aplicada, mayor es la generación de energía eléctrica del sistema (MR Contreras, MS Delgado, 2013).

Algunas investigaciones se han llevado a cabo con base en este procedimiento, concluyendo que las celdas de combustible orgánico se pueden utilizar como un biosensor para determinar en corto tiempo la $\mathrm{DBO}_{5}$ de muestras de aguas residuales. 
Posterior a lo anotado, se realizó la modificación de la concentración inicial del electrolito pasando de $1.0 \mathrm{M}$ a 2.0, 3.0 y luego a 4.0 M. Para esto se utilizó una resistencia de $200 \Omega$ en el circuito externo. Con lo evaluado, se obtuvo que la ausencia de un electrolito restringe la generación de energía eléctrica. Con agua destilada y sin ningún electrolito en ésta, se alcanzaron valores mínimos de voltaje y de corriente: $24 \mathrm{mV}$ y 0.04 mA. Así mismo, con la incorporación de $\mathrm{NaCl}$ como electrolito $(1.0 \mathrm{M})$, se observó un aumento considerable en estos valores, llegando hasta 420 $\mathrm{mV}$ y $3.2 \mathrm{~mA}$ respectivamente.

Las variaciones posteriores de la concentración del electrolito no representaron una variación tan considerable como lo fue el cambio de agua destilada a una solución de $\mathrm{NaCl}$ de $1.0 \mathrm{M}$. Con la última concentración ensayada $\left(\begin{array}{lll}4.0 & \mathrm{M}\end{array}\right)$, se obtuvieron valores máximos de voltaje y corriente de $800 \mathrm{mV}$ y $4.2 \mathrm{~mA}$ respectivamente (Fig. 9).

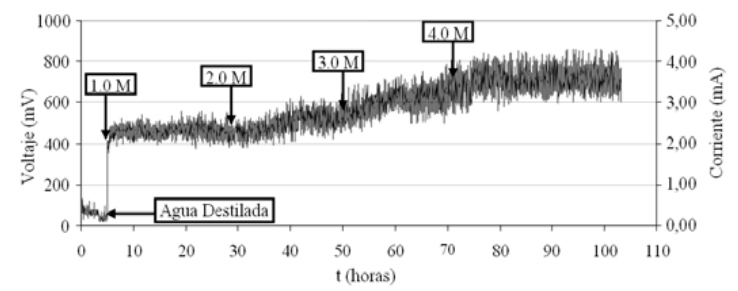

Fig. 9. Voltaje y corriente generada para diferentes concentraciones del electrolito $(\mathrm{NaCl})$. Fuente: El autor.

Contrario a lo recomendado por diversos autores, en el sistema configurado el ánodo no estaba en contacto directo con los microorganismos de la cámara de oxidación. En este sentido, ellos debían adherirse a la membrana aniónica, facilitando así la transferencia de sus electrones al medio donde se encontraba el electrolito, cuya función principal era la de aumentar el área de transferencia de dichos electrones al ánodo.

Luego de realizado esto, se volvió a colocar una concentración del electrolito de $1.0 \mathrm{M}$, y se procedió a evaluar el efecto que tenía la variación de la concentración de $\mathrm{FeCl}_{3}$ contenido en la cámara de reducción. Esto fue realizado utilizando la resistencia de $200 \Omega$.

En un principio, se ensayó sólo con agua destilada sin la presencia de $\mathrm{FeCl}_{3}$ en la cámara de reducción. Posteriormente, se varió la concentración de $0.1 \mathrm{M}$ a $0.2 \mathrm{M}$ (Fig. 10).
Cuando se adicionó el cloruro férrico al agua destilada de la cámara de reducción su pH bajó a 2.0 unidades. Este $\mathrm{pH}$ fue ajustado posteriormente a 9.7 con la adición de bicarbonato de sodio $\left(\mathrm{Na}_{2} \mathrm{HCO}_{3}\right)$. Con esto, se obtuvo un aumento en los valores de corriente y voltajes, los cuales se elevaron paulatinamente durante 13 horas aproximadamente. Luego de la modificación del $\mathrm{pH}$ se alcanzaron valores promedios de corriente y voltaje de $2.7 \mathrm{~mA}$ y $554 \mathrm{mV}$ respectivamente. Cuando se aumentó la concentración de cloruro férrico a $0.2 \mathrm{M}$, los valores de corriente y voltaje tendieron a bajar durante 2 horas, tiempo después del cual se volvió a ajustar el $\mathrm{pH}$, y posteriormente permanecieron constantes.

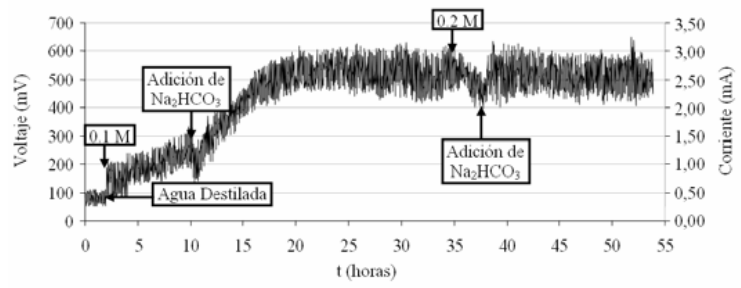

Fig. 10. Voltaje y corriente generada para diferentes concentraciones de $\mathrm{FeCl}_{3}$. Fuente: $\mathrm{El}$ autor.

Luego de lo realizado, se restituyó la concentración inicial de $\mathrm{FeCl}_{3}(0.1 \mathrm{M})$ en la cámara de reducción y se redujo el área del cátodo para operar el sistema con diferentes relaciones de áreas ánodo/cátodo. Fue así como se pudieron evaluar distintas relaciones de áreas 1:1, 1:2, 1:3 y 1:4.

Se obtuvo que para la relación de áreas de 1:4 se alcanzaron los mayores valores de corriente y voltaje de esta variación de la configuración 3.84 mA y $784 \mathrm{mV}$ respectivamente (Fig. 11).

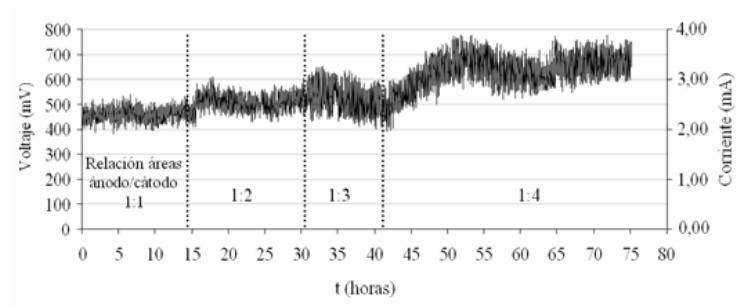

Fig. 11. Voltaje y corriente generada para diferentes relaciones de áreas ánodo/cátodo. Fuente: El autor.

Este procedimiento se hizo con el fin de probar la hipótesis de que al tener el cátodo una menor área, es más fácil que los electrones provenientes de la cámara de oxidación entren en contacto con el $\mathrm{FeCl}_{3}$ al tener un recorrido menor sobre éste. De 
esta manera, se registraron los valores de los voltajes y se procedió a determinar la corriente en forma similar a los ensayos anteriores, con la resistencia de $200 \Omega$.

Luego de realizado lo anterior, se varió la resistencia del circuito sucesivamente de 10, 50, 100, 200, 500 a 1000 ohmios, previa estabilización del voltaje con el circuito abierto. Con la resistencia de $10 \Omega$ se obtuvieron los mayores valores de corriente del sistema: 5.71 mA. Para 50, $100,200,500$ y $1000 \Omega$ se obtuvieron valores promedios de corriente de 3.57, 2.67, 2.09, $0.75 \mathrm{y}$ $0.34 \mathrm{~mA}$ respectivamente (Fig. 12).

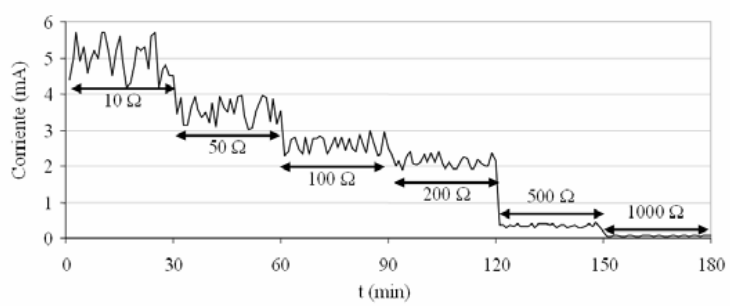

Fig. 12. Corriente generada para diferentes resistencias. Fuente: El autor.

Los resultados de este ensayo tienen este comportamiento decreciente, puesto que, al ir cambiando la resistencia del sistema en el cual se hacía una dosificación constante y continúa del combustible (agua residual), la carga de la fuente tiende a bajar pero nunca a agotarse.

Con los datos de corriente obtenidos, se determinó la carga generada en cada una de las tres configuraciones evaluadas en la Fase II (Fig. 13).

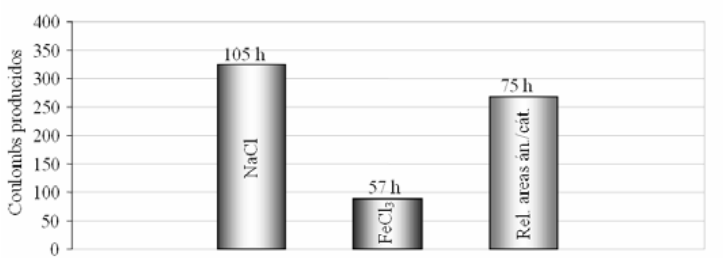

Fig. 13. Carga generada durante el tiempo de operación en cada una de las configuraciones de la fase II. Fuente: El autor.

Esto demuestra que los parámetros que afectan el desempeño de una celda de combustible orgánico bajo las condiciones operadas son en su orden: la concentración del electrolito, la relación de áreas ánodo/cátodo y por último la concentración de $\mathrm{FeCl}_{3}$.

\section{CONCLUSIONES}

Con la elaboración de esta investigación se comprobó que es viable acoplar una celda de combustible orgánico a un proceso de tratamiento biológico de aguas residuales con el fin de extraer parte de la energía que se genera a nivel microbiano en el proceso de degradación de la materia orgánica, y dar un valor agregado al tratamiento de las aguas residuales.

Se estableció que existe una relación entre la generación de energía eléctrica y el grado de madurez de la población de microorganismos encargados de degradar la materia orgánica. A mayor madurez de los microorganismos mayor será su capacidad de generar electrones para transferir a un circuito exterior. Bajo este esquema, las celdas de combustible orgánico pueden ser utilizadas para predecir valores del contenido de materia orgánica presentes en cuerpos de agua.

Se comprobó que no es necesario que los microorganismos estén en contacto directo con el ánodo para que haya transferencia de electrones a éste, por esta razón es viable utilizar una membrana aniónica y junto a ella un electrolito para aumentar el área de transferencia de los electrones al ánodo.

Se determinó que, en su orden, los factores que tienen incidencia en la generación de energía eléctrica son: la concentración del electrolito, la relación de áreas ánodo/cátodo y por último la presencia de un aceptor final de electrones diferente al oxígeno en la cámara de reducción.

Poder generar energía eléctrica a partir de un producto de desecho como lo son las aguas residuales crea un reto interesante a futuro sí se tiene en cuenta que los combustibles fósiles, principales fuentes de energías usadas hoy en día en todo el mundo, generan tantos efectos adversos al medio ambiente.

\section{RECONOCIMIENTO}

El autor expresa sus agradecimientos al ingeniero Sergio Barrera Tapias y a la Universidad de Los Andes, Bogotá D.C., por los recursos facilitados en la realización de este trabajo.

\section{REFERENCIAS}

Logan, B. E., \& Elimelech, M. (2012). Membranebased processes for sustainable power 
generation using water. Nature, 488(7411), 313-319.

L.O. T. Tellez, M. P. S. Delgado, W. M. R.Contreras. (2014). Modelo de interventoría de tecnologías de información en el área de conocimiento de la gestión del alcance de pmbok ${ }^{\circledR}$ y alineado con iso 21500 y cobit ${ }^{\circledR}$. REVISTA COLOMBIANA DE TECNOLOGÍAS DE AVANZADA, ISSN: 1692-7257. 1(23).

Tangarife Tellez, L., Sánchez Delgado, M., \& Rojas Contreras, W. (2014). Modelo de interventoría de tecnologías de información en el área de conocimiento de la gestión del alcance de pmbok $®$ y alineado con iso 21500 y cobit ${ }^{\circledR}$. REVISTA COLOMBIANA DE TECNOLOGÍAS DE AVANZADA, 1(23).

Metcalff \& Eddy, Inc. 2013, Wastewater Engineering Treatment and Resource Recovery. McGraw Hill.

MR Contreras, MS Delgado, (2013), Arquitectura de software para el servicio de soporte de tecnología de información basada en servicios web Revista Colombiana de Tecnologías de Avanzada ISSN: 1692-7257.

Minteer, S. D. (2017). Methods in Biological Fuel Cells. In Springer Handbook of Electrochemical Energy (pp. 743-755). Berlin, Heidelberg: Springer Berlin Heidelberg.

Pasternak, G., Greenman, J., \& Ieropoulos, I. (2017). Self-powered, autonomous Biological Oxygen Demand biosensor for online water quality monitoring. Sensors and Actuators B: Chemical, 244, 815-822.

Roustazadeh Sheikhyousefi, P., Nasr Esfahany, M., Colombo, A., Franzetti, A., Trasatti, S. P., \& Cristiani, P. (2017). Investigation of different configurations of microbial fuel cells for the treatment of oilfield produced water. Applied Energy, 192, 457-465.

Santoro, C., Arbizzani, C., Erable, B., \& Ieropoulos, I. (2017). Microbial fuel cells: From fundamentals to applications. A review. Journal of Power Sources, 356, 225-244.

Sieniutycz, S., \& Jezowski, J. (2017). Energy optimization in process systems and fuel cells.

YE Santafé, BD Chaparro..., (2013), Deteccción de patrones característicos con transformadas wavelet en señales electromiográficas del cuádriceps Revista Colombiana de Tecnologías de Avanzada, ISSN: 1692-7257. 